\title{
The attainment of herd immunity with a restructured risk of contact infection in compartmental models
}

Kian Boon Law ( $\boldsymbol{\nabla}$ kblaw@crc.gov.my )

Institute for Clinical Research, Malaysia https://orcid.org/0000-0002-1175-1307

Kalaiarasu M Peariasamy

Institute for Clinical Research, Malaysia

Hishamshah Ibrahim

Office of the Director General, Ministry of Health Malaysia

Noor Hisham Abdullah

Office of the Director General, Ministry of Health Malaysia

\section{Research Article}

Keywords: Herd immunity, infectious disease, vaccination, COVID-19, deterministic model

Posted Date: March 2nd, 2021

DOI: https://doi.org/10.21203/rs.3.rs-289776/v1

License: (9) This work is licensed under a Creative Commons Attribution 4.0 International License.

Read Full License 


\title{
The attainment of herd immunity with restructured risk of contact infection in compartmental model
}

\author{
Law Kian Boon ${ }^{1}$, Kalaiarasu M. Peariasamy², Hishamshah Ibrahim³ \& Noor Hisham Abdullah³ \\ ${ }^{1}$ Digital Health Research and Innovation Unit, Institute for Clinical Research, National Institutes of Health, \\ Ministry of Health Malaysia \\ ${ }^{2}$ Institute for Clinical Research, National Institutes of Health, Ministry of Health Malaysia \\ ${ }^{3}$ Office of the Director General, Ministry of Health Malaysia
}

\begin{abstract}
Introduction:

The risk of contact infection among susceptible individuals in a randomly mixed population can be reduced by the presence of immune individuals and this concept is referred as herd immunity. Although herd immunity is observed in vaccinated population for some infectious diseases, it has never been truly attained in compartmental models such as the susceptible-infectious-recovered (SIR) model. This paper introduces a new SIR framework to overcome the limitation of the conventional SIR model in attaining the herd immunity.
\end{abstract}

\section{Methods:}

Two SIR models were newly developed based on the reduced risk of contact infection. The first model A assumes that the risk of contact infection reduces as soon as susceptible individuals are infected and move from class $S(t)$ to $I(t)$, therefore incorporating both prevalence of infectious and susceptible individuals into its force of infection. The second model B assumes the risk of contact infection would reduce after infected individuals have recovered from infection and move from class $I(t)$ to $R(t)$, therefore incorporating both prevalence of infectious and inverse of prevalence of recovered individuals into its force of infection. Then, numerical simulations were applied to obtain approximate solutions for all three conventional SIR model, new SIR model A and model B for comparison under exact and arbitrary conditions with $\beta=0.3$ and $\sigma=0.1$ to mimic the infection dynamics with basic reproduction ratio $\left(r_{0}\right)$ of 3.0 and herd immunity threshold (HIT) of 0.667 (66.7\%).

\section{Results and discussion:}

All three models performed likewise at the initial stage of epidemic. The conventional SIR model simulated the epidemic diminishing when $94.0 \%$ of the population had been infected and recovered, way above its HIT. Model A simulated the epidemic waning when $66.7 \%$ of the population had been infected and recovered, in line with its HIT, however, the model conceptualized the herd immunity incorrectly. Model B simulated the epidemic waning at $75.6 \%$, slightly above its HIT and was in line with the fundamental of herd immunity. The difference between model A and model B can be attributed to the proportion of infectious individuals, and this would increase in infectious disease with high transmissibility. The threshold theorem derived based on $r_{0}$ may not be sufficient for effective control and eradication of infectious disease with high transmissibility like the COVID-19.

\section{Conclusion:}

The newly developed SIR model that includes the inverse of proportion of recovered individuals is more accurate and credible for modelling infection or vaccine induced herd immunity, especially in COVID-19.

Keywords: Herd immunity, infectious disease, vaccination, COVID-19 


\section{Introduction}

Herd immunity became a fixture of epidemiology in 1930s, and took on fresh prominence in 1950s and 1960s as new vaccines raised crucial questions for public health policy on the proportion of vaccinated population for eradication of infectious disease. ${ }^{1}$ The fundamental of herd immunity is that the risk of contact infection among susceptible individuals can be reduced by the presence and proximity of immune individuals in a randomly mixed population. ${ }^{2}$ Although the impact of herd immunity has been observed in vaccinated population of many periodical childhood epidemics, such as measles, mumps, rubella, pertussis, chickenpox, and polio for decades, it has never been truly attained in mathematical models.

Mathematical models such as deterministic models are widely used to study infection dynamics, especially in the COVID-19 pandemic. These models use flexible compartmental framework and robust assumptions for a wide range of indications. ${ }^{3-6}$ The susceptible-infectious-recovered (SIR) model classifies individuals by epidemiological status, based on their ability to host and transmit a pathogen. ${ }^{7}$ This simplifies the manifestation of infection dynamics for most infectious diseases. Besides compartmentalization, the SIR model also assumes complete immunity is conferred by a single infection, hence encompassing the epidemiological notion of herd immunity, in which an indirect protection among susceptible individuals may stem from the rising proportion of recovered and immune individuals, either through infection or vaccination. ${ }^{2,8,9}$

On the basis of declining transmissibility, the herd immunity threshold (HIT) can be estimated from the basic reproduction ratio $\left(r_{0}\right)$ of SIR model to guide the eradication program for target epidemic through vaccination. ${ }^{2}$ However, the conventional SIR model simulates an epidemic with $r_{0}$ of 3.0 waning when $94 \%$ of a population have been infected and recovered with immunity, far beyond the HIT of $67 \%$ for control or eradication of epidemic. The dissociation between model forecast and HIT exposes the fundamental drawback of using the conventional SIR model in modelling vaccine induced herd immunity, especially in the COVID-19 pandemic. As many COVID-19 models have been configured based on the conventional SIR framework during the COVID-19 pandemic, the vagueness of the conventional SIR framework in understanding the system dynamics and modelling vaccine induced herd immunity is of practical and exceptional concern now. ${ }^{10-14}$ This study introduces and evaluates a newly developed SIR model to overcome the ambiguity of the conventional SIR model in describing and attaining the transmisssion dynamics of infectious disease in a randomly mixed population.

\section{Materials and methods}

\section{The Kermack \& McKendrick's SIR model.}

Kermack \& McKendrick postulated the first SIR deterministic model for infectious disease in 1927, on the basis that an infectious disease spreads from affected to unaffected by contact infection. ${ }^{9}$ The SIR model then became the fundamental of most infectious disease models developed since. In the SIR model, contact infection refers to the direct or indirect act of contracting pathogen, with risk of contact infection given by the proportion of infectious individuals in a population. Besides, the SIR model also assumes that the an infectious disease would grow exponentially at the beginning and eventually diminishes due to the depleted number of susceptible individuals. ${ }^{7}$ 


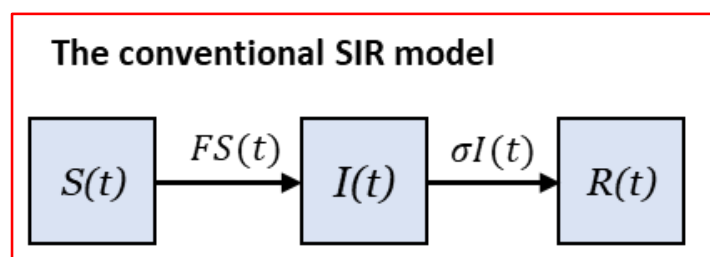

Force of infection, $F=\beta I(t) / N$
Figure 1: The conventional SIR model developed by Kermack \& McKendrick (1927). Individuals move from class $S(t)$ to $I(t)$ at a rate proportional to $\beta, \frac{I(t)}{N}$, and $S(t)$. The proportion of infectious individuals signifies the risk of contact infection among susceptible individuals.

The conventional SIR model divides a homogenous population, $N$ into three distinct classes: susceptible denoted by $S(t)$, infectious denoted by $I(t)$ and recovered denoted by $R(t)$. Susceptible are individuals at equal risk of contracting an infection. Infectious are infected individuals who have developed infectivity and can transmit pathogens like virus when come into contact with susceptible individuals. Recovered are individuals who have recovered from infection and acquired immunity. As the SIR model assumes recovered individuals are no longer susceptible to infection, hence, recovered individuals are also regarded "removed" from the infection dynamics (Figure 1). In brief, the conventional SIR model features a partially mixed population, in which the transmission rate can only be affected by the presence of infectious individuals, but not recovered individuals.

In the SIR model, the transition of individuals between classes occurs at a rate proportional to the number of individuals in the respective classes, and constant coefficients of transmission and recovery. For instance, individuals move from class $S(t)$ to $I(t)$ at a transition rate proportional to the number of susceptible individuals, $S(t)$, transmission coefficient $(\beta)$ and risk of contact infection, given by the proportion of infectious $\frac{I(t)}{N}$. The product of $\beta$ and $\frac{I(t)}{N}$ denotes the force of infection, $F$ of an infectious disease. After being infected, individuals would start to recover and move from class $I(t)$ to $R(t)$ at a transition rate proportional to the number of infectious individuals, $I(t)$ and recovery coefficient $(\sigma)$, given by the reciprocal of infection duration.

Mathematically, the conventional SIR model functions in a set of coupled nonlinear ordinary differential equations (ODEs) that deterministically describes the slopes or flows between classes over time as follows:

$$
\begin{aligned}
& \frac{d S(t)}{d t}=-\frac{\beta I(t) S(t)}{N}, \\
& \frac{d I(t)}{d t}=\frac{\beta I(t) S(t)}{N}-\sigma I(t), \\
& \frac{d R(t)}{d t}=\sigma I(t) .
\end{aligned}
$$

Without vital dynamics, the population size is given by:

$$
N=S(t)+I(t)+R(t)
$$

Equation (2) can be converted into prevalence or proportion by dividing each notation with $N$ :

$$
1=\frac{S(t)}{N}+\frac{I(t)}{N}+\frac{R(t)}{N}
$$


The inverse of proportion of recovered individuals is then given by:

$$
1-\frac{R(t)}{N}=\frac{S(t)}{N}+\frac{I(t)}{N}
$$

At the initial stage of an epidemic, both the number of infectious and recovered individuals are very small as compared to the population size, therefore, $\frac{I(t)}{N} \approx 0, \frac{R(t)}{N} \approx 0$ and $\frac{S(t)}{N} \approx 1$. At the end stage of an epidemic, the number of infectious individuals becomes very small again, therefore, $1-\frac{R(t)}{N} \approx \frac{S(t)}{N}$.

\section{The basic reproduction ratio, $\boldsymbol{r}_{\mathbf{0}}$}

According to Equation (1-2) of the conventional SIR model. At the initial stage of an epidemic, $\frac{S(t)}{N} \approx$ 1. Then, we would obtain the following equation:

$$
\frac{d I(t)}{d t}=(\beta-\sigma) I(t)
$$

The integral of Equation (5) is an exponential function as follow:

$$
I(t)=I_{0} e^{(\beta-\sigma) t}
$$

Equation (6) indicates a crucial state that determines the spread of an infectious disease at its initial stage. An infectious disease would spread exponentially if:

$$
\begin{gathered}
\beta-\sigma>0, \\
\frac{\beta}{\sigma}-1>0, \\
\frac{\beta}{\sigma}>1 .
\end{gathered}
$$

The ratio between $\beta$ and $\sigma$ denotes the basic reproduction ratio $\left(r_{0}\right)$ of an infectious disease, which is also defined as the number of secondary cases caused by a single primary case in a wholly susceptible population. ${ }^{15,16}$ An infectious disease would become epidemic when its $r_{0}$ is bigger than 1 . Otherwise, it would diminish. The herd immunity threshold (HIT) of an epidemic can be derived from its $r_{0}$ with the following equation:

$$
\mathrm{HIT}=1-\frac{1}{r_{0}}
$$

The HIT marks the level at which the proportion of susceptible individuals has to be vaccinated to control or eradicate an infectious disease. ${ }^{17}$

\section{The risk of contact infection in a randomly mixed population}

In a randomly mixed population, the risk of contact infection could be affected by the proportion of susceptible individuals, infectious individuals and recovered individuals, concurrently. For instance, the 
rising proportion of infectious individuals would increase the risk of contact infection. On the other hand, the rising proportion of recovered individuals or declining proportion of susceptible would decrease the risk of contact infection as well. In order to understand how proportion of susceptible or recovered individuals can affect the infection dynamics in a randomly mixed population, two models were developed based on assumptions below:

Assumption A: The risk of contact infection would reduce as soon as susceptible individuals are infected and moved from class $S(t)$ to class $I(t)$. In this circumstance, the risk of contact infection is given by both proportion of susceptible individuals, $\frac{S(t)}{N}$ and infectious individuals, $\frac{I(t)}{N}$. By incorporating both proportions into Equation (1-1) to (1-3), we obtain a new set of ODEs as follow:

$$
\begin{aligned}
\frac{d S(t)}{d t} & =-\frac{\beta I(t)[S(t)]^{2}}{N^{2}}, \\
\frac{d I(t)}{d t} & =\frac{\beta I(t)[S(t)]^{2}}{N^{2}}-\sigma I(t), \\
\frac{d R(t)}{d t} & =\sigma I(t) .
\end{aligned}
$$

The product of $\beta, \frac{I(t)}{N}$ and $\frac{S(t)}{N}$ denotes the new force of infection, $F_{A}$ of an infectious disease in the new SIR model A.

Assumption B: The risk of contact infection would reduce after infected individuals have recovered and moved from class $I(t)$ to class $R(t)$. In this circumstance, the risk of contact infection is given by the proportion of infectious individuals, $\frac{I(t)}{N}$ and the inverse of proportion of recovered individuals, $1-\frac{R(t)}{N}$ or $\frac{N-R(t)}{N}$. By incorporating both proportion and inverse of proportion into Equation (1-1) to (1-3), we obtain another new set of ODEs as follow:

$$
\begin{aligned}
& \frac{d S(t)}{d t}=-\frac{\beta I(t)[N-R(t)] S(t)}{N^{2}}, \\
& \frac{d I(t)}{d t}=\frac{\beta I(t)[N-R(t)] S(t)}{N^{2}}-\sigma I(t), \\
& \frac{d R(t)}{d t}=\sigma I(t) .
\end{aligned}
$$

The product of $\beta, \frac{I(t)}{N}$ and $\frac{N-R(t)}{N}$ denotes the new force of infection, $F_{B}$ of an infectious disease in the new SIR model B. The inverse of proportion of recovered individuals was used to signify the reduced risk of contact infection.

According to Equation (11-2) and (12-2), at the initial stage of an epidemic when proportions of infectious and recovered individuals approach zero, both model $\mathrm{A}$ and $\mathrm{B}$ would generate the same $r_{0}$ and HIT values as of the conventional SIR model. Hence, all three models are equally accurate for describing the initial dynamics of transmission when proportions of infectious and recovered individuals approach zero. However, both model A and B would surpass the conventional SIR model at the later part of epidemic when the proportion of recovered individuals can no longer be ignored. 


\section{Model simulation and sensitivity analyses}

Firstly, we applied numerical simulations to obtain approximate solutions for all three models under the exact and arbitrary condition with $\beta=0.3$ and $\sigma=0.1$ to mimic infection dynamics with $r_{0}=3.0$ and $\mathrm{HIT}=0.667$. In the arbitrary condition, $66.7 \%$ infected population were required for herd or population immunity to take effect for control or eradication of epidemic. At the end of epidemic, the proportion remained susceptible in the population was expected to be $33.3 \%$, given by the inverse of HIT. Next, we calculated differences between the conventional and new SIR models for susceptible, infectious, total infection (I Total), new Infection (I New) and recovered over time $t$. In sensitivity analyses, we evaluated the total infections and recovered individuals achieved across $r_{0}$ ranging from 1.1 to 4.0 in all three models. Numerical simulations were performed in R version 3.6.3 with "deSolve" package. Graphics were organized in Microsoft Excel 2019. Modelling outputs were presented in graphs with proportion of population (\%) as the $y$-axis and arbitrary time $t$ as the $x$-xis.

\section{Ethics requirement}

The study was registered with National Medical Research Register. No ethics approval was required.

\section{Results:}

The new SIR model retained most of the underlying assumptions except for one major transformation, that was the rate of transmission can also be affected by the prevalence of susceptible individuals (model A) or prevalence recovered individuals (model B) in a randomly mixed population. This assumption can be achieved by incorporating the proportion of susceptible individuals (model $A$ ) or the inverse of proportion of recovered individuals (model B) into force of infection of SIR model (Figure 2).

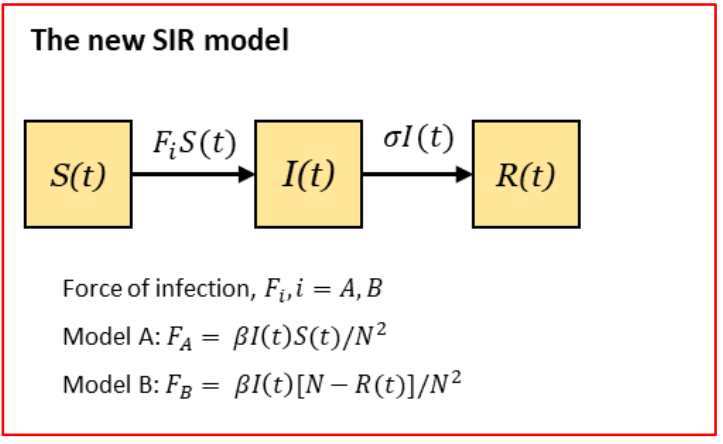

Figure 2: The compartmental structure of new SIR models and force of infections in a randomly mixed population

At the initial stage of epidemic, when both number of infectious and recovered individuals were very small compared to the population size, proportions of infectious and recovered individuals approach zero, and all three models performed likewise. Therefore, infection dynamics of all three models can be described accurately by the $r_{0}$. Moreover, the HIT derived using the $r_{0}$ value thus applied across all three models. Figure 3 presents results of numerical simulations under the exact $r_{0}$ of 3.0 and HIT of $66.7 \%$ for all three models, respectively.

Expectedly, the conventional SIR model simulated the epidemic waning at a level way above the HIT. According to Figure 3A, the epidemic only diminished when $94.0 \%$ of the population had been infected and recovered with immunity, $27.3 \%$ above the HIT, consequent to not accounting the reduced risk of contact infection over time. Surprisingly, the new SIR model A simulated the epidemic waning when both total infections and recovered individuals reach the HIT of $66.7 \%$ (Figure 3B). The new SIR model B 
simulated the epidemic waning when $75.6 \%$ of the population had been infected and recovered with immunity, $8.9 \%$ above the HIT (Figure 3C).

In line with the fundamental of herd immunity, reduced risk of contact infection protected more susceptible individuals from contracting infection. Hence, both new models generated less new infections than the conventional SIR model over time (Figure 3D). The risk of contact infection reduced tremendously after $t=20$ when total infected individuals reached 6 to $7 \%$ and total recovered individuals reached 2 to $3 \%$ of population in both new SIR models (Figures 3E and 3F). The result suggested that the herd immunity could have induced protective effect among susceptible even at the early stage of epidemic.

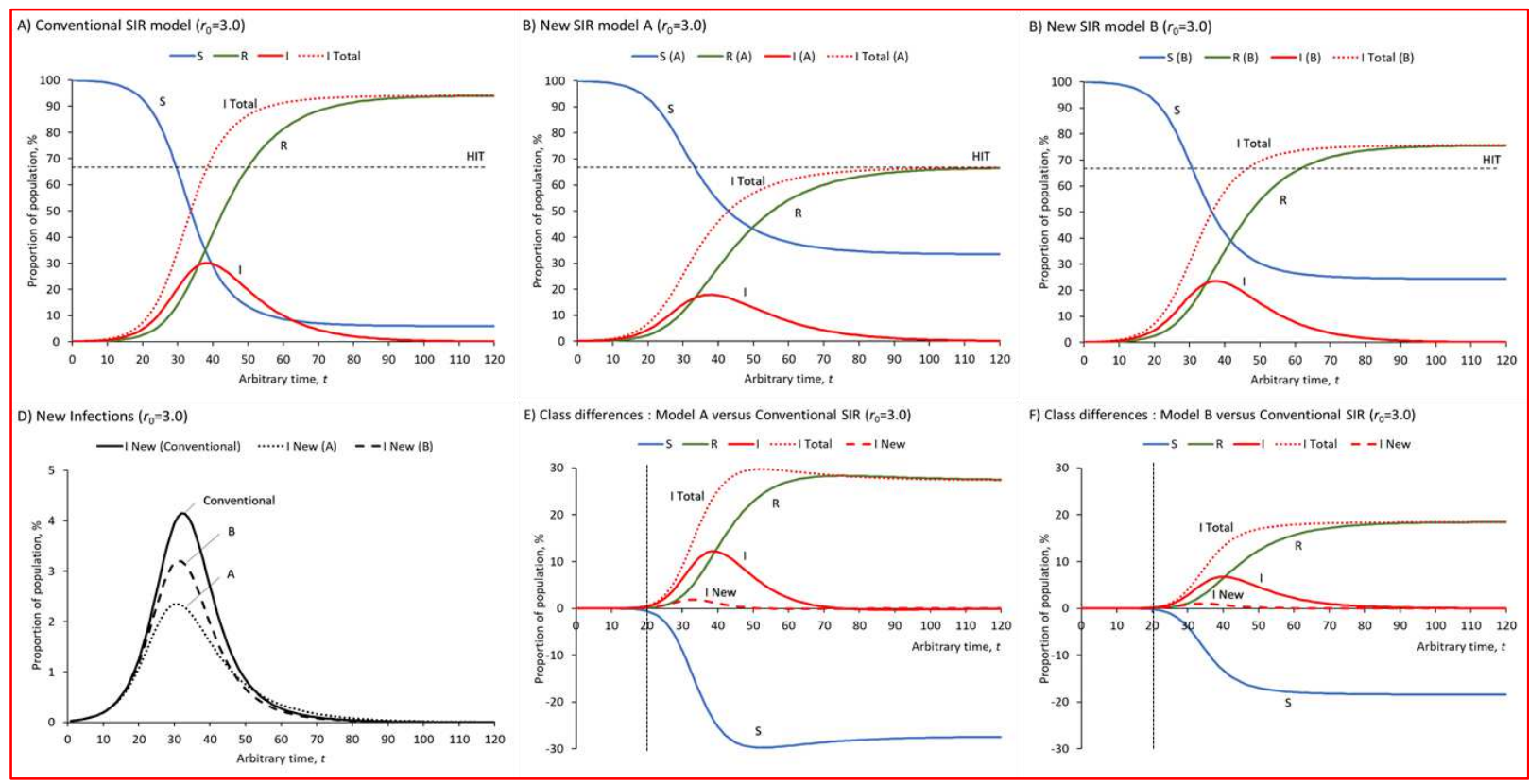

Figure 3: The infection dynamics of both conventional and new SIR models with different risk frameworks of contact infection.

Part A, B and C illustrate the infection dynamics simulated by the conventional SIR model, new SIR model A and B. The HIT is marked by horizontal dotted black line in part A, B and C. Part D presents new infections generated by the conventional SIR model, and new SIR model A and B. Part E presents class differences between conventional SIR model and new SIR model A. Part F presents class differences between conventional SIR model and new SIR model B. The time point where herd immunity starts to take effect is marked by the vertical dotted black line in part $E$ and $F$.

The growth of epidemic was successfully suppressed due to the reduced risk of contact infection in both model $A$ and $B$. The number of total infections and recovered individuals with immunity had been brought down to the HIT derived from the $r_{0}$, especially for model A (Figure 4). As model A assumed reduced risk of contact infection right after susceptible were infected, hence, least total and new infections were generated in simulation. Unfortunately, model A did not conceptualize the principle of herd immunity correctly even its modelling outputs were in line with the HIT precisely. Model B conceptualized the herd immunity as an indirect protection driven by the presence and proximity of immune individuals, which was more welcome. As the recovery process and gaining of immunity requires time, more total infections than the HIT were likely. 


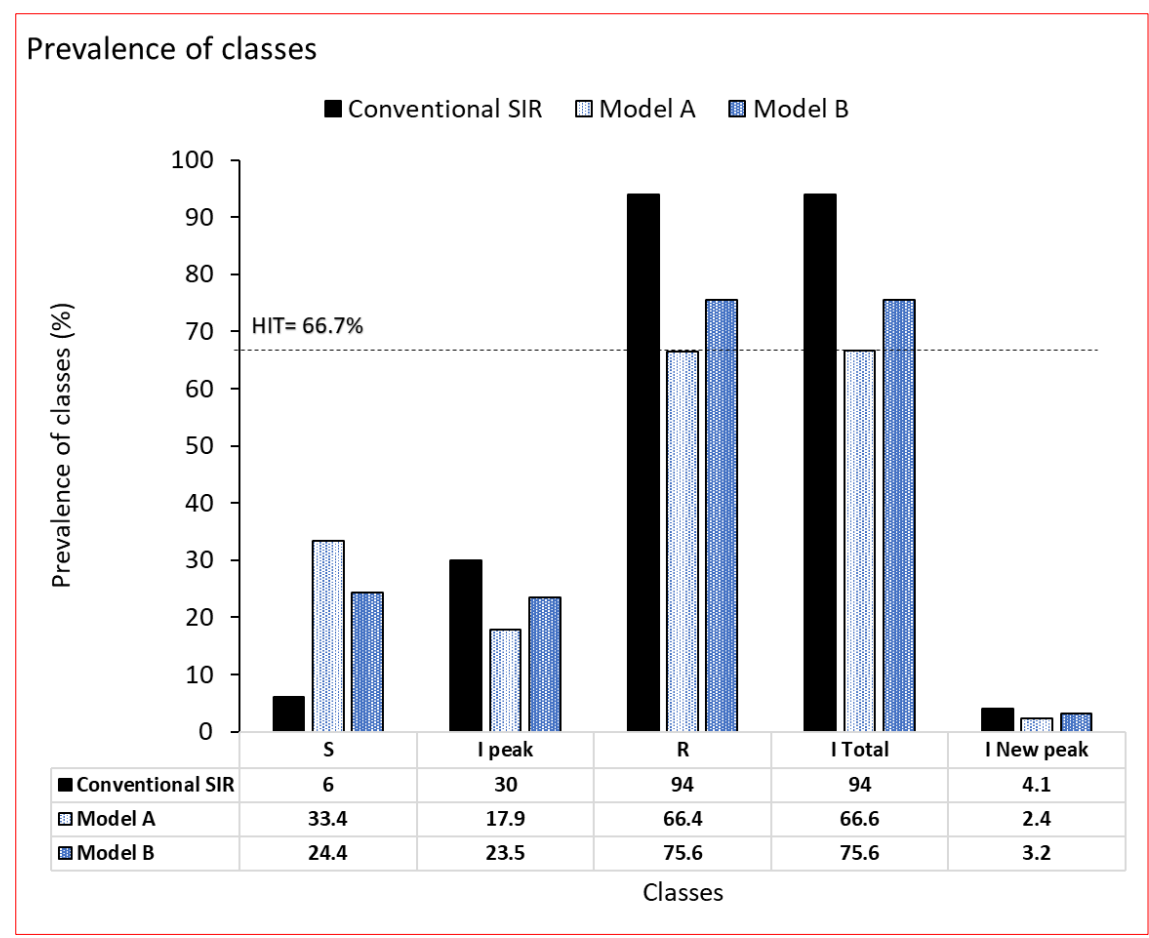

Figure 4: Comparison of proportions of classes achieved in the conventional SIR model and new SIR models. The HIT is marked by the dotted black line.

Sensitivity analyses were conducted for all three models to estimate the threshold for waning of epidemic across $r_{0}$ values ranging from 1.1 to 4.0 (Figure 5). The threshold of epidemic waning for the conventional SIR model ranged from $16.9 \%$ to $98.0 \%$ across $r_{0}$ values from 1.1 to 4.0 , consistently above the HIT. The threshold of epidemic waning for the new SIR model A ranged from $9.1 \%$ to $75.0 \%$ across $r_{0}$ values from 1.1 to 4.0, in line with the HIT precisely. Remarkably, the threshold of epidemic waning for the new SIR model B ranged from 9.7\% to $85.9 \%$ across $r_{0}$ values from 1.1 to 4.0. The threshold of epidemic waning for both model $\mathrm{A}$ and $\mathrm{B}$ were similar when the $r_{0}$ was small, and increased as the $r_{0}$ became larger. 


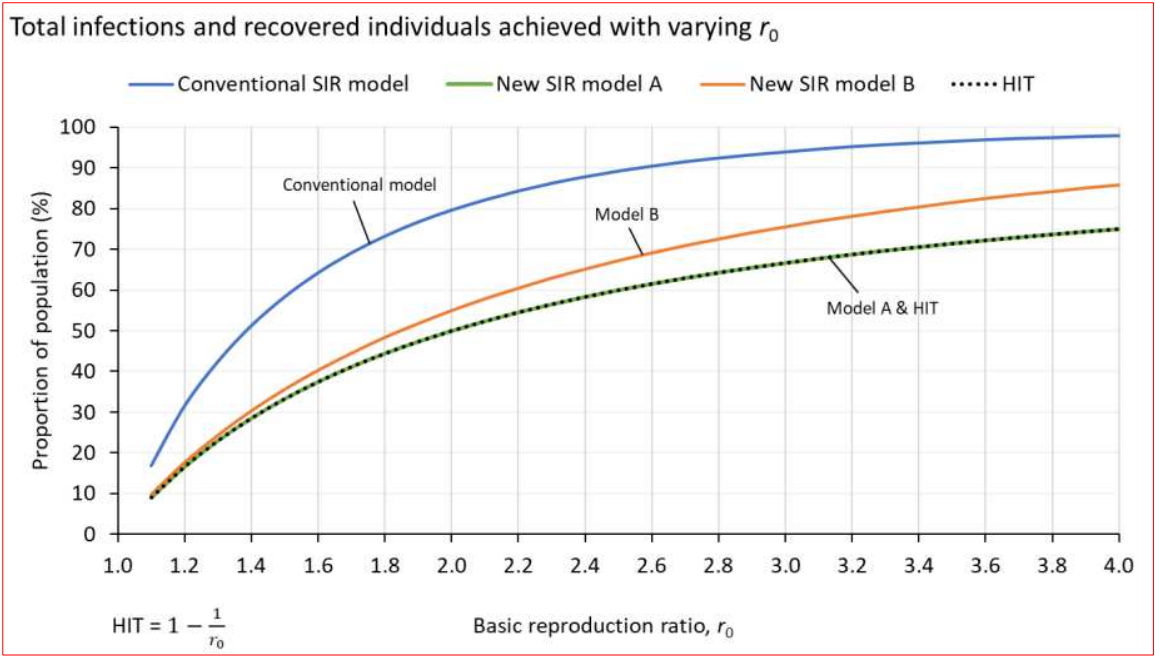

Figure 5: Total infections and recovered individuals simulated by the conventional SIR model, new SIR model $A$ and $\mathrm{B}$.

Both new and conventional SIR models were similar in many aspects, but also differed in some aspects due to the new risk determinant of contact infection. Table 1 summarizes similarities and differences between the conventional SIR model and new SIR models.

Table 1: similarities and differences between conventional SIR model and new SIR models.

\section{Similarities}

- Compartmental structure.

- Homogenous population.

- Complete immunity is conferred by a single infection.

- Transition rate proportional to the number of individuals in the respective classes, constant coefficients of transmission and recovery, and risk of contact infection.

- The $r_{0}$ can be estimated at the initial stage of infection.

\section{Differences}

Conventional SIR model

- Partially mixed population

- The risk determinant of contact infection is the proportion of infectious individuals.

- The risk of contact infection changes according to proportion of infectious individuals.

- Epidemic vanishes as a result of the exhausted number of susceptible.

- Epidemic wanes at the threshold way above the HIT calculated based on $r_{0}$.
New SIR model A

- Randomly mixed population

- The risk determinant of contact infection are proportions of infectious and susceptible individuals.

- The risk of contact infection declined as soon as susceptible individuals are infected.

- Epidemic vanishes as a result of the exhausted number of susceptible and declined risk of contact infection.

- Epidemic wanes precisely at the HIT calculated based on its $r_{0}$.
New SIR model B

- Randomly mixed population

- The risk determinant of contact infection are proportion of infectious individuals and inverse of proportion of recovered individuals.

- The risk of contact infection declined after infectious individuals have recovered from infection.

- Epidemic vanishes as a result of the exhausted number of susceptible and declined risk of contact infection.

- Epidemic wanes at the threshold close to the HIT calculated based on its $r_{0}$ when 
- $F=\frac{\beta I(t)}{N}$

- More total and new infections, and less susceptible at the end of epidemic.

- Herd immunity is not attained in modelling output.

- Not in line with the principle of herd immunity at all.
- $F_{A}=\frac{\beta I(t) S(t)}{N^{2}}$

- Less total and new infections, and more susceptible at the end of epidemic.

- Herd immunity is attained in modelling output.

- In line with the principle of herd immunity correctly. the $r_{0}$ is small, and deviate from the HIT when the $r_{0}$ becomes bigger

- $F_{B}=\frac{\beta I(t)[N-R(t)]}{N^{2}}$

- Less total and new infections, and more susceptible at the end of epidemic, depending on the transmissibility of infectious disease.

- Herd immunity is attained in modelling output.

- In line with the principle of herd immunity correctly.

\section{Discussion}

The drawback of the conventional SIR model can be attributed to the removal of the impact of recovered and immune individuals from the system dynamics of infectious disease. As a result, the conventional SIR model overestimated the infection dynamics and generated more infections at the end of simulation. This drawback has rendered the conventional SIR model inappropriate as the framework for modelling infectious diseases in a randomly mixed population. Our simulations showed that the conventional SIR model overestimated the total infections by 10 to $30 \%$ above the HIT across $r_{0}$ values ranging from 1.1 to 4.0 at the end of epidemic.

Our simulations also showed the HIT theorem can only be realized accurately by incorporating the proportion of susceptible individuals into the model, that is to assume that the risk of contact infection reduces as soon as susceptible individuals are infected and move from class $S(t)$ to $I(t)$. According to the threshold theorem proposed by Dietz in 1975, the HIT was derived from a vaccine model developed based on the conventional SIR framework, therefore did not inherit the reduced risk of contact infection in a randomly mixed population. ${ }^{18}$ Hence, the HIT theorem may not be sufficient for estimating the level of herd or population immunity required for eradication and control of epidemic. Besides, the HIT may not be sufficient too for infectious disease with high transmissibility, as high transmissibility may lead to high proportion of infectious individuals over time. Therefore, the use of HIT calculated from the $r_{0}$ may result in suboptimal control of epidemic, especially in COVID-19.

The simulated outcome of model A was plausible, however violating the fundamental of herd or population immunity featuring an indirect protection caused by the presence or proximity of immune individuals in a randomly mixed population. ${ }^{2}$ The model B overcame this problem by incorporating the inverse of proportion of recovered individuals into its force of infection, $F_{B}$, and successfully suppressed the infection dynamics at increasing thresholds across $r_{0}$ values ranging from 1.1 to 4.0. Hence, model B provides a more credible framework for modelling infectious disease in a randomly mixed population with realistic immunity thresholds for control and eradication of epidemic.

A noteworthy finding was that the herd immunity may take effect even at the early stage of epidemic, in which the proportion of recovered and immune individuals may not be high. This finding may explain the sharp fall of COVID-19 cases in countries like United Kingdom (UK), Indonesia and Germany, shortly after the rollout of vaccine, and countries like India even before the rollout of vaccine. ${ }^{19}$ With the 
sharp fall of COVID-19 cases after the rollout of vaccines, Britain has initiated its unlocking process guided by the COVID-19 trajectory. ${ }^{20}$ While many countries are still struggling with the COVID-19 unrest, the sharp fall of COVID-19 cases in India since September 2020 without vaccines has caught the global attention. Many researchers attributed the decline of COVID-19 cases without vaccination in India to its population herd immunity and younger demographic. A national serological survey conducted by the Indian Council of Medical Research (ICMR) revealed that up to $21 \%$ or 290 million of adult population in India have been exposed to the COVID-19 virus and developed immunity against the virus. ${ }^{21}$

As the world is still under attack by the COVID-19 pandemic, the use of deterministic models featuring a reduced risk of contact infection consequent to the rising prevalence of recovered and immune individuals, either as a result of infection or vaccination would provide a more reliable framework to understand the epidemiology and impact of eradication strategies in the COVID-19 pandemic. Having said that, the attainment of herd immunity in the new SIR model does not imply that the herd immunity can be achieved in real life without taking other critical factors, such as population heterogeneity and weakened population immunity due viral mutations into account.

As of this writing, more than 100 million individuals have been infected by the novel coronavirus with death toll of approximate 2.5 million. ${ }^{19}$ Due to exhausted healthcare resources and increasing asymptomatic manifestations, many infected individuals are not diagnosed and captured into the COVID19 statistics. At the same time, many countries have started mass vaccination program with hope to end the COVID-19 nightmare through vaccine induced population or herd immunity, with target threshold derived from the $r_{0}$ of COVID-19. Vaccine models developed based on the conventional framework could have resulted in misleading findings. Hence, applying the right vaccination threshold and framework in modelling COVID-19 and vaccine induced herd immunity is exceptionally relevant and critical now.

\section{Conclusion}

The paper offers a new framework for SIR model and its variant model in modelling infectious disease in a randomly mixed population. The newly developed SIR model that includes the inverse of proportion of recovered individuals is more accurate and credible for modelling infection or vaccine induced herd immunity, especially in COVID-19. The threshold theorem derived from the $r_{0}$ of infectious disease may not be sufficient for optimal control and eradication of infectious diseases with high transmissibility.

\section{Acknowledgement}

We would like to thank the Director General of Health Malaysia for his permission to publish this article. We would like to thank Associate Professor Dr. Jane Labadin and Dr. Kuan Pei Xuan for proofreading the article and providing constructive inputs before publication.

\section{Competing interests}

The authors declare no competing interests.

\section{Contributions}

K.B.L and K.M.P conceived and planned the study.

K.B.L contributed to the design of the compartmental model and simulation.

K.M.P, H.S and N.H.A supervised the implementation of the study.

Data analysis and graphics were done by K.B.L.

All authors contributed to the interpretation of the findings and approved the final version for publication.

\section{References}


1. Jones, D. \& Helmreich, S. A history of herd immunity. The Lancet 396, 810-811 (2020).

2. Fine, P., Eames, K. \& Heymann, D. L. "Herd Immunity": A Rough Guide. Clin. Infect. Dis. 52, 911-916 (2011).

3. Chowell, G., Sattenspiel, L., Bansal, S. \& Viboud, C. Mathematical models to characterize early epidemic growth: A review. Phys. Life Rev. 18, 66-97 (2016).

4. Peak, C. M., Childs, L. M., Grad, Y. H. \& Buckee, C. O. Comparing nonpharmaceutical interventions for containing emerging epidemics. Proc. Natl. Acad. Sci. 114, 4023-4028 (2017).

5. Mandal, S., Sarkar, R. R. \& Sinha, S. Mathematical models of malaria--a review. Malar. J. 10, 202-202 (2011).

6. Law, K. B. et al. Tracking the early depleting transmission dynamics of COVID-19 with a time-varying SIR model. Sci. Rep. 10, 21721 (2020).

7. Keeling, M. J. \& Danon, L. Mathematical modelling of infectious diseases. Br. Med. Bull. 92, 33-42 (2009).

8. Metcalf, C. J. E., Ferrari, M., Graham, A. L. \& Grenfell, B. T. Understanding Herd Immunity. Trends Immunol. 36, 753-755 (2015).

9. Kermack, W. O., McKendrick, A. G. \& Walker, G. T. A contribution to the mathematical theory of epidemics. Proc. R. Soc. Lond. Ser. Contain. Pap. Math. Phys. Character 115, 700-721 (1927).

10. Bartsch, S. M. et al. Vaccine Efficacy Needed for a COVID-19 Coronavirus Vaccine to Prevent or Stop an Epidemic as the Sole Intervention. Am. J. Prev. Med. 59, 493-503 (2020).

11. Bubar, K. M. et al. Model-informed COVID-19 vaccine prioritization strategies by age and serostatus. Science eabe6959 (2021) doi:10.1126/science.abe6959.

12. Sandmann, F., Davies, N., Vassall, A., Edmunds, W. J. \& Jit, M. The potential health and economic value of SARS-COV-2 vaccination alongside physical distancing in the UK: transmission model-based 
future scenario analysis and economic evaluation. medRxiv 2020.09.24.20200857 (2020)

doi:10.1101/2020.09.24.20200857.

13. Kwok, K. O., Lai, F., Wei, W. I., Wong, S. Y. S. \& Tang, J. W. T. Herd immunity - estimating the level required to halt the COVID-19 epidemics in affected countries. J. Infect. 80, e32-e33 (2020).

14. Scherer, A. \& McLean, A. Mathematical models of vaccination. Br. Med. Bull. 62, 187-199 (2002).

15. Heffernan, J. M., Smith, R. J. \& Wahl, L. M. Perspectives on the basic reproductive ratio. J. R. Soc. Interface 2, 281-293 (2005).

16. Siettos, C. I. \& Russo, L. Mathematical modeling of infectious disease dynamics. Virulence 4, 295306 (2013).

17. Anderson, R. M. \& May, R. M. Vaccination and herd immunity to infectious diseases. Nature 318, 323-329 (1985).

18. Dietz K. Transmission and control of arbovirus diseases. In: Ludwig D, Cooke KL, eds. Epidemiology. Pheladelphia PA: Society for Industrial and Applied Mathematics, 1975: 104-21.

19. Johns Hopkins University Coronavirus Resources Center. https://coronavirus.jhu.edu/.

20. Peter Walker. Step by step: how England's Covid lockdown will be lifted. The Guardian.

21. ICMR sero survey: One in five Indians exposed to Covid-19. BBC News (2021). 
Figures

\section{The conventional SIR model}

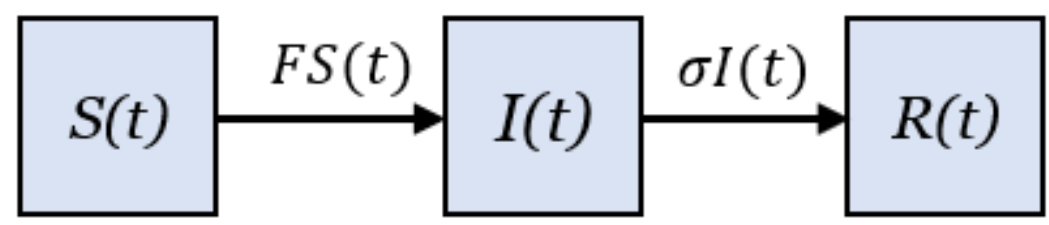

Force of infection, $F=\beta I(t) / N$

\section{Figure 1}

The conventional SIR model developed by Kermack \& McKendrick (1927). Individuals move from class

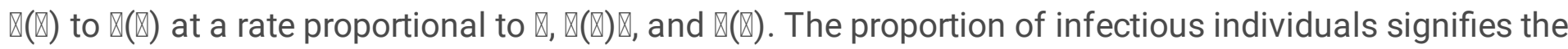
risk of contact infection among susceptible individuals.

\section{The new SIR model}

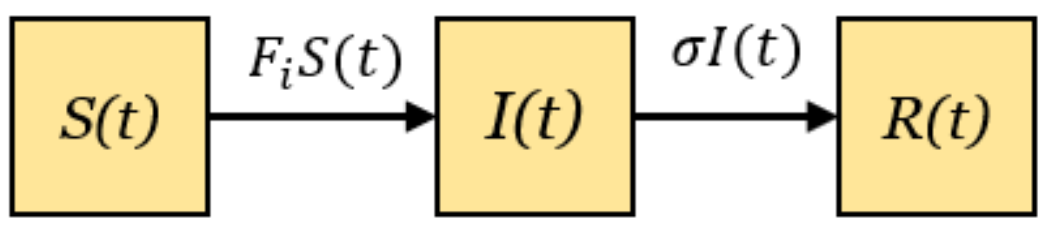

Force of infection, $F_{i}, i=A, B$

Model A: $F_{A}=\beta I(t) S(t) / N^{2}$

Model B: $F_{B}=\beta I(t)[N-R(t)] / N^{2}$

\section{Figure 2}

The compartmental structure of new SIR models and force of infections in a randomly mixed population 
A) Conventional SIR model $\left(r_{0}=3.0\right)$ $\longrightarrow \mathrm{S}-\mathrm{R}-\mathrm{I} \cdots \cdots \cdot$ Total

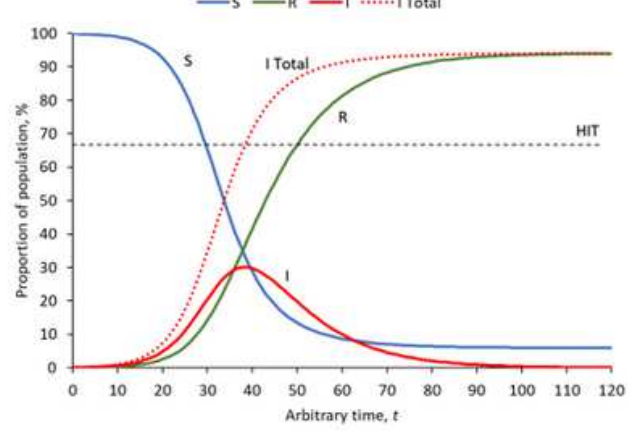

D) New Infections $\left(r_{0}=3.0\right)$

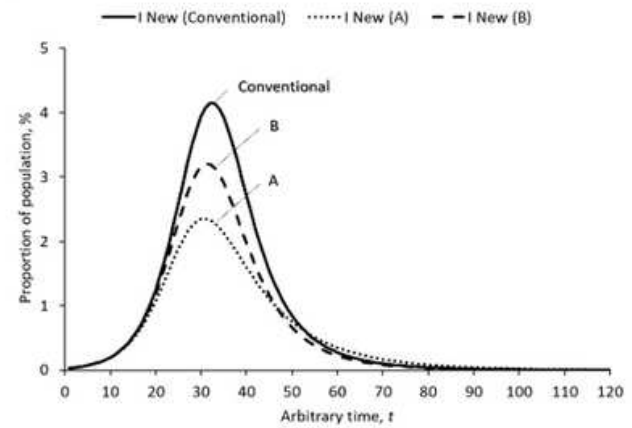

B) New SIR model A $\left(r_{0}=3.0\right)$

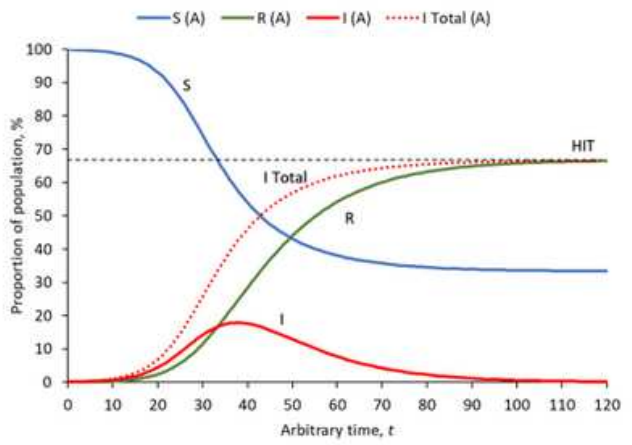

E) Class differences : Model A versus Conventional SIR $\left(r_{0}=3.0\right)$

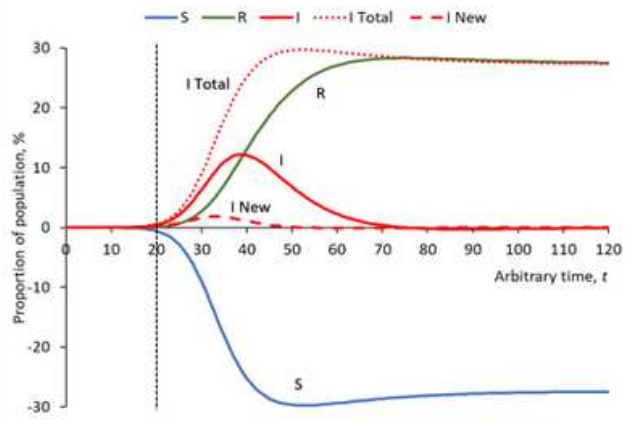

B) New SIR model B $\left(r_{0}=3.0\right)$

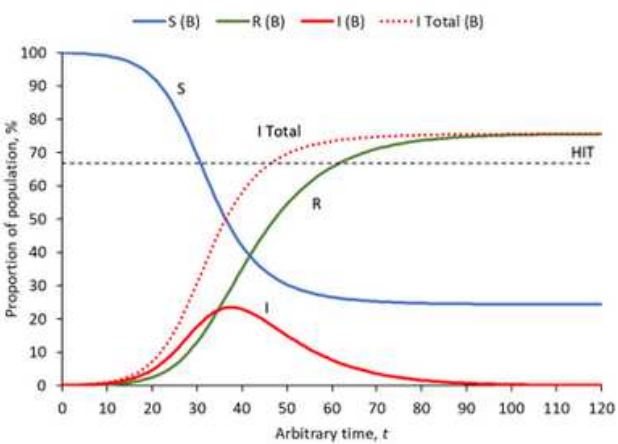

F) Class differences : Model $B$ versus Conventional SIR $\left(r_{0}=3.0\right)$

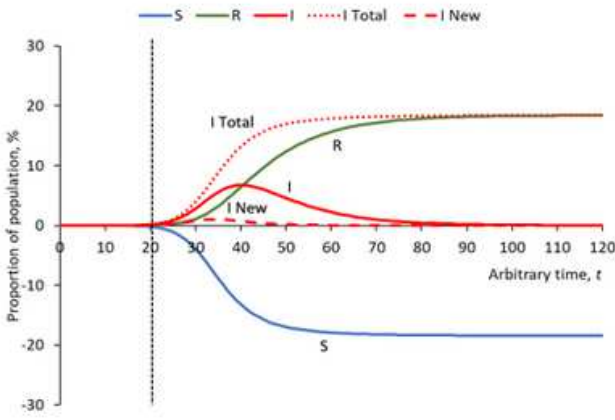

\section{Figure 3}

The infection dynamics of both conventional and new SIR models with different risk frameworks of contact infection. Part A, B and C illustrate the infection dynamics simulated by the conventional SIR model, new SIR model A and B. The HIT is marked by horizontal dotted black line in part A, B and C. Part $D$ presents new infections generated by the conventional SIR model, and new SIR model A and B. Part E presents class differences between conventional SIR model and new SIR model A. Part F presents class differences between conventional SIR model and new SIR model B. The time point where herd immunity starts to take effect is marked by the vertical dotted black line in part $E$ and $F$. 
- Conventional SIR T Model A Model B

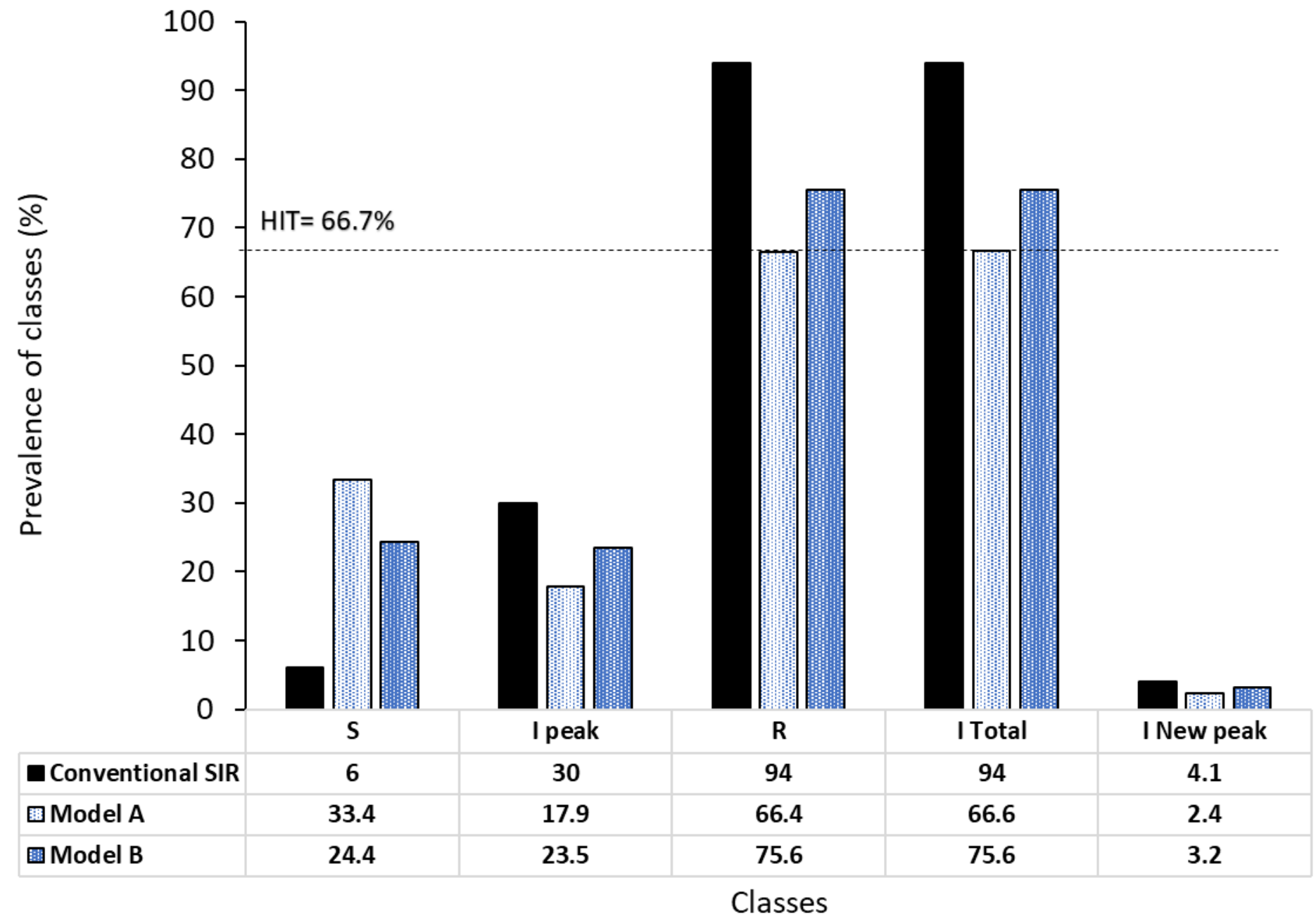

Figure 4

Comparison of proportions of classes achieved in the conventional SIR model and new SIR models. The HIT is marked by the dotted black line. 
Total infections and recovered individuals achieved with varying $r_{0}$

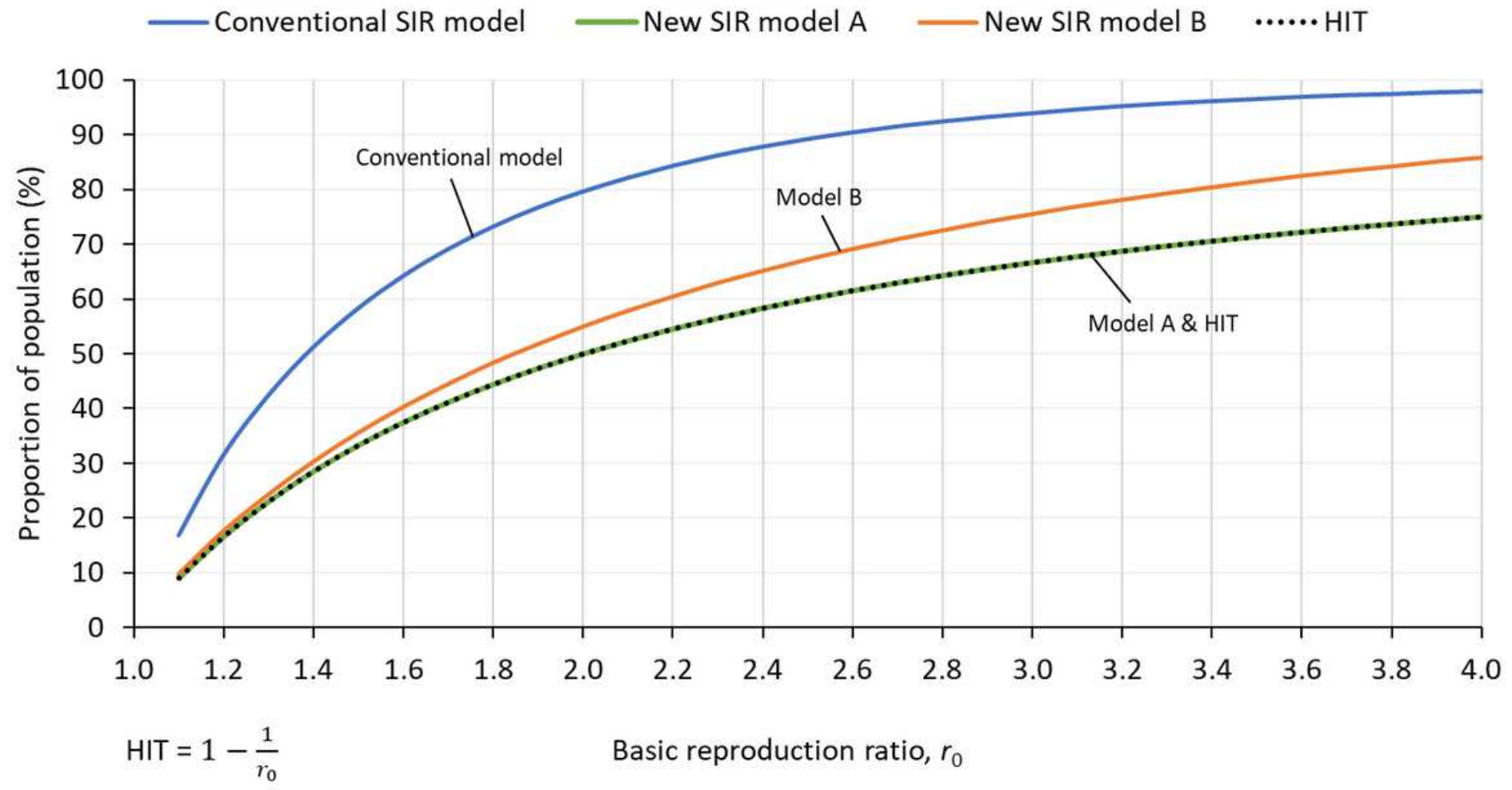

Figure 5

Total infections and recovered individuals simulated by the conventional SIR model, new SIR model A and $\mathrm{B}$. 